\title{
New evidence for the zoom lens model using the RSVP technique
}

\author{
MARIA I. BARRIOPEDRO \\ European University of Madrid, Madrid, Spain \\ and \\ JUAN BOTELLA \\ Universidad Autónoma de Madrid, Madrid, Spain
}

\begin{abstract}
A main prediction from the zoom lens model for visual attention is that performance is an inverse function of the size of the attended area. The "attention shift paradigm" developed by Sperling and Reeves (1980) was adapted here to study predictions from the zoom lens model. In two experiments two lists of items were simultaneously presented using the rapid serial visual presentation technique. Subjects were to report the first item he/she was able to identify in the series that did not include the target (the letter T) after he/she saw the target. In one condition, subjects knew in which list the target would appear; in another condition, they did not have this knowledge, having to attend to both positions in order to detect the target. The zoom lens model predicts an interaction between this variable and the distance separating the two positions where the lists are presented. In both experiments, this interaction was observed. The results are also discussed as a solution to the apparently contradictory results with regard to the analog movement model.
\end{abstract}

The ability to selectively attend to a spatial location that does not correspond to the line of sight is well known. The study of this phenomenon has given rise to proposals about the spatial and/or temporal limitations of this selection process. Eriksen and Hoffman (1972) found evidence supporting the idea that attention can operate as a focus of about $1^{\circ}$ of visual angle. From the probabilitycuing paradigm used by Posner and his colleagues (Posner, 1978, 1980; Posner, Snyder, \& Davidson, 1980) emerged the idea that the application of spatial attention is analogous to shining a spotlight on the visual field. From this point of view, the spotlight metaphor, the focus of visual attention is limited in spatial extent, can illuminate only a small region of the visual world at any one moment, is temporally limited in its movements, and moves in an analog manner along surfaces (Egly \& Homa, 1991; Shulman, Remington, \& McLean, 1979; Tsal, 1983). However, the evidence quite clearly suggests that attention is able to be distributed over large areas of the visual field, as well as operating in a focused mode (Erik-

This research has been partially supported by the Dirección General de Investigación Cientifica y Técnica of Spain, Project PB94-0157. A preliminary report of the present research was presented at the conference Converging Operations in the Study of Visual Selective Attention (Barriopedro, 1994). The authors wish to thank James Juola, Adam Reeves, Jan Theeuwes, and an anonymous reviewer for their helpful comments and suggestions, which greatly improved the structure and language of the preliminary version of the manuscript. Correspondence should be addressed to J. Botella, Facultad de Psicología, Universidad Autónoma de Madrid, Cantoblanco s/n, 28049 Madrid, Spain (e-mail: juan.botella@uam.es). sen \& St. James, 1986; Eriksen \& Yeh, 1985; Jonides, 1980, 1983; LaBerge, 1983; LaBerge \& Brown, 1986). Moreover, two of the major sources of evidence for analog movements of attention (Shulman et al., 1979; Tsal, 1983) have been questioned on several counts (Eriksen \& Murphy, 1987; Yantis, 1988).

Alternative frameworks have also been proposed to account for the properties of spatial selectivity of visual attention. Eriksen and his colleagues (Eriksen, Pan, \& Botella, 1993; Eriksen \& Rohrbaugh, 1970; Eriksen \& St. James, 1986; Eriksen \& Yeh, 1985; Murphy \& Eriksen, 1987) have provided evidence suggesting that attentional focus is variable in size. The zoom lens model proposed by these authors implies that, as the focus is narrowed, the attentional resources concentrate on a small area, and as the focus is widened, they become diffused over a wider area. As a result, there is an inverse relationship between size of attentional focus and efficiency of processing. The attentional focus is not sharply demarcated from the remainder of the visual field but instead is characterized by a gradient of decreasing processing efficiency. The change of locus of attention would seem to be discrete, with the time required for shifts from one location to another being independent of the distance between the locations (Eriksen \& Webb, 1989; Murphy \& Eriksen, 1987). From this resource-based conception of attention, attentional allocation consists in the gradual but rapid buildup of resources at the location of the attended object. The independence between distance and time for shifting is a feature shared by other theories, such as the episodic theory (Sperling \& Weichselgartner, 1995), in which the term quantal is used, as opposed to analog. 
According to the meridian boundary model (Hughes \& Zimba, 1985, 1987), attention must be directed to large regions of the visual field, and it cannot be directed to specific locations. Attentional effects are produced by the activation of an attended region and/or the suppression of an unattended region, where regions are visual hemifields (Hughes \& Zimba, 1985) or visual quadrants (Hughes \& Zimba, 1987) bounded by and defined in terms of the vertical and/or horizontal visual meridians. However, many studies have yielded evidence contrary to the notion of hemifield activation (Downing \& Pinker, 1985; Klein \& McCormick, 1989; McCormick \& Klein, 1990; Shulman, Sheehy, \& Wilson, 1986; Shulman, Wilson, \& Sheehy, 1985).

Downing and Pinker (1985) suggested that the allocation of attention to a spatial location produces a gradient of attention, with the peak centered in that location and whose density decreases with increasing distance from that location. In this account, there is no need to assume a moving spotlight of attention. Distance effects are due to the gradient of attentional resources. Downing (1988) explored the distribution of costs and benefits for different kinds of tasks and found that costs are a function of distance in detection and brightness discrimination tasks, but not in those of form and orientation discrimination. Downing asserted that, in the latter cases, there is also an attentional gradient, but with so sharp a slope that it works virtually as described in spotlight models.

Shaw and Shaw (1977) and Shaw (1978) proposed a much more flexible system. This model conceives of visual attention as consisting of a fixed amount of resources that can be distributed over different discrete areas of the display. Processing of stimuli in these locations would proceed in parallel, but at different rates if the allocation of resources to the different positions is unequal. However, this account is in disagreement with the results obtained by other researchers (Eriksen \& Yeh, 1985; Posner, Nissen, \& Ogden, 1978) suggesting that attention cannot be simultaneously focused on separate areas in the visual field (see also General Discussion).

After reviewing the empirical base of the main spacebased allocation models of selective attention, we believe that a more apt analogy is that of a zoom lens, as proposed by Eriksen and his colleagues (Eriksen \& St. James, 1986; Eriksen \& Yeh, 1985; Murphy \& Eriksen, 1987). In this paper, we will try to extend this model to tasks and situations different from those usually employed, in order to test its generalizability. The bulk of research in the field is based on the cuing paradigm with short stimulus onset asynchronies (SOAs), where the cues can yield some automatic attraction of attention that is added to the attraction due to its informative value. The effect of abrupt-onset stimuli (Yantis, 1995; Yantis \& Johnston, 1990; Yantis \& Jones, 1991; Yantis \& Jonides, 1984, 1990 ) is well known, as is the fact that attention is captured by any salient stimulus (Theeuwes, 1995), so it seems advisable to use experimental paradigms in which these effects are controlled. We have attempted to do so in the experiments described below by using the rapid serial visual presentation (RSVP) technique.

It also seemed interesting to study how the zoom lens model can account for subjects' performance in tasks different from those usually employed in this context, in order to increase its credibility and to explore the boundaries of the analogy. With this goal, we designed some experiments combining spatial selection with the RSVP technique, in which a series of stimuli is presented in a single spatial location at a high rate. This technique has been used to study selective attention based on nonlocation criteria, such as shape, color, case of letters, or identity of letters or words (Botella \& Eriksen, 1992; Botella, Garcia, \& Barriopedro, 1992; Eriksen \& Spencer, 1969; Gathercole \& Broadbent, 1984; Kanwisher, 1987; Lawrence, 1971; McLean, Broadbent, \& Broadbent, 1983; Raymond, Shapiro, \& Arnell, 1992; Shapiro, Raymond, \& Arnell, 1994). In the present experiments, as in our previous work (Barriopedro, 1994) we presented two series of items. The task was to identify the first item in one of the series after detecting the target (the letter $\mathrm{T}$ ) in the other series. The first version of this task was developed by Sperling and Reeves (1980; Reeves \& Sperling, 1986) for the study of attention reaction time (i.e., the time taken to shift attention). A main feature of the experiments by Sperling and Reeves was that the target list included only letters and the response series included only numbers.

Here, the main manipulation was previous information concerning in which list the target would be presented. We call this variable spatial certainty. The rationale was that if the subject knows that the T will appear on the left (position-known condition), then he/she will use a small focus over that position, and, after detecting the $T$, the focus will be moved to the other position. On the other hand, if the subject does not know where the target will be presented (position-unknown condition), he/she will be forced to use a wider focus that encompasses both positions in order to avoid missing the target. To foster the use of these attentional strategies, we included both letters and digits in both lists, instead of one being made up of only letters and the other of only digits.

The second manipulation was the distance between the spatial positions where the series were presented. When the distance was increased, subjects had to enlarge the focus in the position-unknown condition, but not in the position-known condition. In the latter condition, what was increased was the distance to be covered between the two positions where attention was focused during a trial.

\section{EXPERIMENT 1}

The zoom lens model has three main features (Eriksen, 1990; Eriksen \& Murphy, 1987; Eriksen \& St. James, 1986; Eriksen \& Yeh, 1985): (1) the size of the attentional focus is variable, (2) processing efficiency is inversely related to the size of the attended area, and (3) the rate of accumulation of resources in a specific position is independent of the distance between that position and 
the position where they were previously concentrated. It is fairly obvious that the three features are basically independent, above all Point 3. We did not test specific points of the model; rather, we tested the model as a whole, deriving predictions for specific tasks.

We presented two rapid series of stimuli, one at each side of the fixation point. The subject was asked to report the first item he/she was able to identify in the series that did not include the target after he/she saw the target; the target was always the letter $\mathrm{T}$. We manipulated the spatial certainty about the target in such a way that, in one condition, the subject knew before the trial in which series the $\mathrm{T}$ would be included, but not in the other (positionknown, PK, and position-unknown, PU, respectively). Furthermore, the distance between the two series of stimuli was manipulated. The manipulation of the spatial certainty was intended to force the subjects to use different attentional dispositions at the beginning of the trial.

Assuming the zoom lens model, we expected that the subjects in the PK condition, given the difficulty of the task, would probably concentrate their attention on the position where the series including the target was presented and, once the target was detected, shift to the position of the other series. In the PU condition, the subjects attended to both series simultaneously to avoid missing the target, applying foci of different sizes depending on the distance between the positions of the series. Consequently, following the assumptions of the zoom lens model, we expected that, in the PK condition, the subjects might respond more slowly as the distance between the series increased, but the only factor responsible for this effect would be the different eccentricity associated with each distance used in the task. This is because we assume that the rate of accumulation of resources at a given position is independent of the distance to the position where those resources where previously concentrated. The same basic conclusion was reached by Reeves and Sperling (1986), who described this regarding their attention gating model: "the gating process observed here is much more suggestive of a faucet-gate theory, in which a gate is opened and closed first at one location and then at another, with no particular dependence on the distance between the locations" (p. 201). The same conclusion has recently been reached by Sperling and Weichselgartner (1995). On the other hand, in the PU condition, in which the subject attended simultaneously to the positions where each series was presented, we expected an effect of the distance. Responses should be slower as the distance increases, since the area to which the subject must attend increases with the distance, and, thus, there will be a smaller amount of resources per surface unit. This effect is added to that of eccentricity. Thus, the expectation was that the effect of distance would differ according to the spatial certainty. We defer to the General Discussion section a more detailed explanation of the mechanism that relates the amount of attention allocated with performance in this task, as reflected by the delay of the items reported.
From the zoom lens model, a significant interaction of the distance between the positions and the spatial certainty was predicted (i.e., the decrease in performance with distance would be sharper in the PU condition than in the PK condition).

\section{Method}

Subjects. Fourteen undergraduate students from the Universidad Autónoma de Madrid with normal or corrected-to-normal vision participated in the experiment.

Apparatus. The experiment was run with an IBM-PC-compatible computer. Eye movements were monitored by a video camera. The subjects who showed obvious eye movements were excluded from the experiment (only 2 were excluded because of apparent eye movements). A chinrest was used to control the distance from the eyes to the screen $(60 \mathrm{~cm})$.

Series of stimuli. The stimuli used to build the series were all uppercase consonants (except $\mathrm{N}$ ) and the digits $1-9$ (size $0.29^{\circ}$ wide $\times 0.67^{\circ} \mathrm{high}$ ). Forty-five pairs of lists with 14 items each were constructed. The target was the letter $\mathrm{T}$, and it could be included equiprobably in serial positions $5,6,7$, or 8 of one of the lists of each pair. This list will be called the target list. The other list will be called the response list. The positions of the other stimuli were determined randomly, with the only restriction that, in the pair of lists for each trial, no one stimulus was repeated. In 5 of those pairs of lists, the target was not included. The subjects were informed of this point in order to avoid mechanical responses. A cross was used as the fixation point. The stimuli and the fixation point were presented in black on a gray background. The luminance of the background was $3.0 \mathrm{~cd} / \mathrm{m}^{2}$, and the lights of the room were off.

Procedure. Before each trial, the fixation point was presented on the screen. The trial began when the subject pressed the space bar. The fixation remained visible for $1,500 \mathrm{msec}$, and the pair of lists were then presented, at the same distance from each side of the cross. The stimuli of each list were presented in a single spatial position, for $116 \mathrm{msec}$ each and with no interval between them (exposure time $=\mathrm{SOA}=116 \mathrm{msec})$. Figure 1 shows the scheme of a trial. When the presentation was finished, a response menu appeared that contained all stimuli included in the series plus two extra options, "I don't know" and "no T." The first one was to be used when the subject did not know the response, and the second was to be used when the letter $T$, the target, had been not presented. The subjects' task was to identify the first stimulus they could in the response list once the letter $T$ was detected in the target list.

Two variables were manipulated: the distance between the lists $\left(1.15^{\circ}, 2.34^{\circ}\right.$ and $3.53^{\circ}$ of visual angle-these were distances between the centers of the stimuli; we refer to these distances as short, medium, and large, respectively) and the spatial certainty about the target, with two conditions, PK and PU. Both variables were manipulated as within-subject factors. In the PK condition, subjects ran two blocks of 45 trials each for each distance. In one block, the target list appeared always at the left side of the fixation point; in the other block, it appeared at the right side. This information was communicated to the subjects before each block. In the PU condition, the subjects also ran two blocks of 45 trials for each distance. In this condition, it was randomly determined for each trial at which side the target list would be presented (left or right of the fixation point). Thus, the subjects participated in 12 experimental blocks of 45 trials, 6 for the PK condition and 6 for the PU condition, 2 for each distance, in two experimental sessions of about $1 \mathrm{~h}$. In each session, the subjects participated in only one condition of spatial certainty, which began with two blocks of 45 trials of practice at the medium distance.

With regard to spatial certainty, the order in which the experimental conditions was administered was balanced, so that half of the subjects ran the PK condition in the first session and the PU condition in the second, and the other half ran the conditions in the 


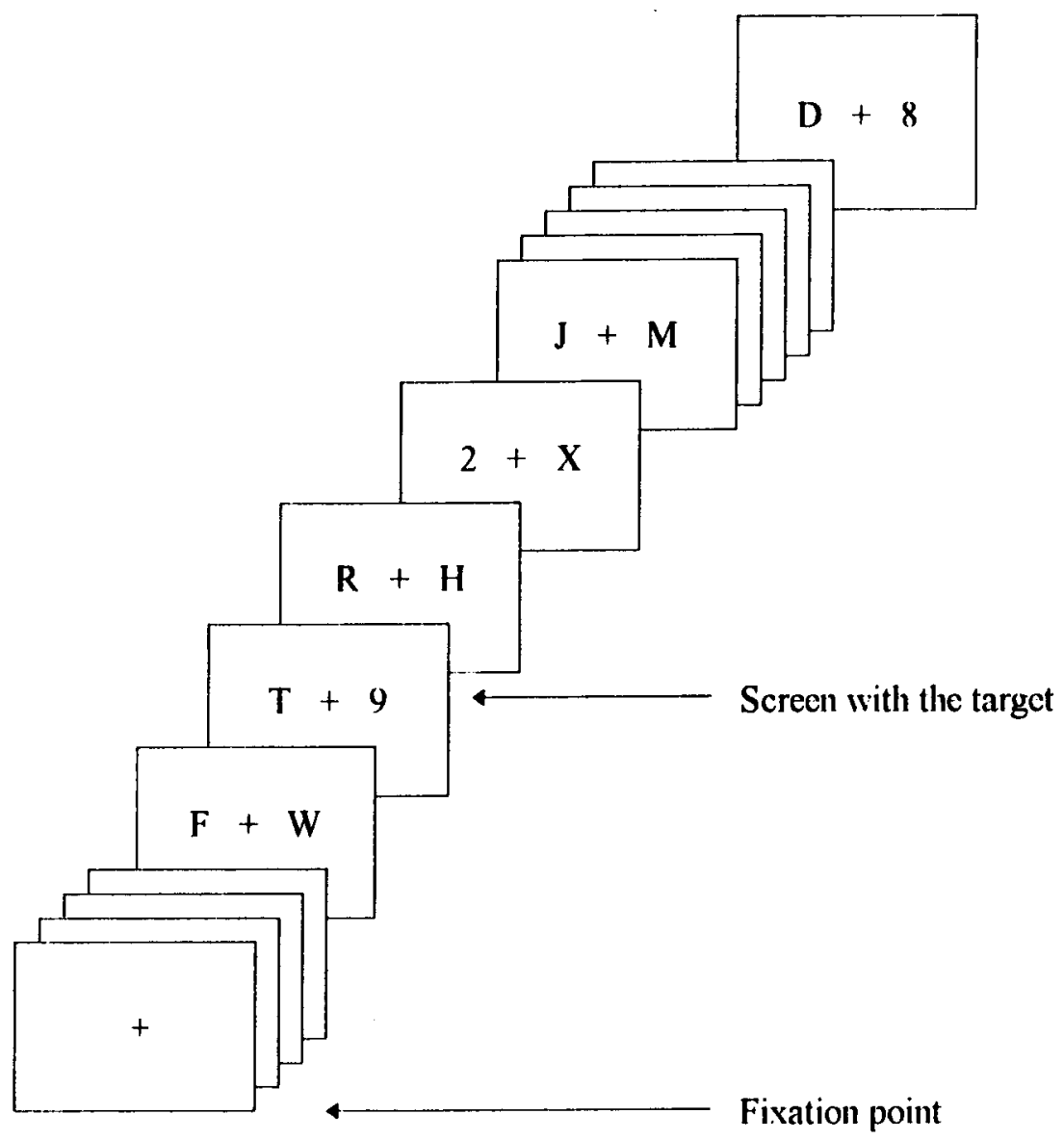

Figure 1. Schematic representation of a trial.

opposite order. The order of the blocks of each distance was determined according to a Latin square.

In the sessions of the PK condition, the order of presentation of the blocks in which the target list was presented at each side of the fixation point was balanced. Thus, for half of the subjects, in the first three experimental blocks, this list was presented at the left and, in the last three blocks, it was presented to the right; for the other half of the subjects, the order was reversed.

Attentional facilitation was operationalized mainly by means of the asynchrony between the $T$ and the item identified. We refer to this main dependent variable as the average serial order of the responses (ASOR). ${ }^{1}$ That is, we assumed that the smaller the attentional facilitation, the later would be the item reported. We have assigned the value 0 to reports corresponding to the stimulus presented simultaneously with the target (in the example of Figure 1, it is the Stimulus 9), 1 to the reporting of the stimulus presented just after the target (in the example, the letter $H$ ), 2 for the next item (the letter $\mathrm{X}$ in the example), and so on. These values were averaged for each subject and experimental condition, in order to obtain the average number of positions that separated the target from the responses, or ASOR. These were the responses considered to be correct. The logic of this measurement procedure is analogous to that of Sperling and his colleagues (Reeves \& Sperling, 1986; Sperling \& Reeves, 1980). The responses corresponding to identi- ties of stimuli presented before the target or after the target but in the target list will not be considered in this paper.

\section{Results}

Given that the preliminary analyses did not show any effect of the position of the target list (left or right of the fixation point), and given that this variable was not relevant for the goals of this research, the data from both sides were collapsed for the rest of the analyses. The data were analyzed by means of a repeated measures $2 \times 3$ analysis of variance (ANOVA), the main factors being spatial certainty (PK, PU) and distance (short, medium, large). Multiple comparisons a posteriori were made with a Tukey test.

In the P.K. condition, the mean values of the ASOR were $3.66,3.79$, and 3.70 for the distances short, medium, and large, respectively. In the PU condition, the values were $3.90,4.23$, and 4.40 , respectively. In the statistical analysis of this variable, the effect of spatial certainty was not significant $[F(1,13)=4.35, p>.05]$, but the effect of distance was significant $[F(2,26)=6.25, p<.01]$. The ASOR with distances large and medium was greater 


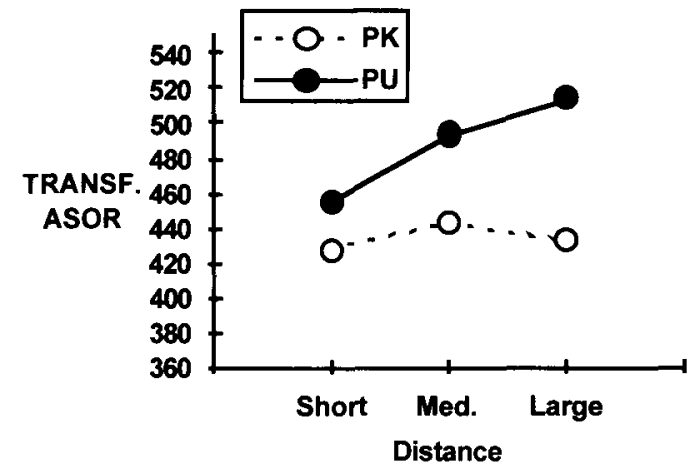

Figure 2. Average serial order of responses (ASOR; in milliseconds) for Experiment 1 (1 item $=116 \mathrm{msec})$.

than with distance short (for the comparison large-short, $p<.01$; for the comparison medium-short, $p<.05$ ). The effect of the interaction of spatial certainty and distance was significant $[F(2,26)=3.80, p<.05]$. The differences found as a function of distance were not significant for the PK condition (for the three comparisons, $p s>.05$ ). For the PU condition, the ASOR was significantly greater for the distances large and medium than for the distance short ( $p \mathrm{~s}<.01$ and .05 , respectively), whereas there was no difference between the distances large and medium $(p>.05)$. Figure 2 shows these results, after transforming the ASOR to milliseconds (taking into account that one item is equivalent to $116 \mathrm{msec}$ ).

\section{Discussion}

The pattern of results fits with the predictions derived from the zoom lens model, which receives additional credibility from an experimental paradigm different from that usually employed for testing it. Apparently, the subjects adapted the size of the attentional focus to the task demands using, in the PK condition, a small focus centered on the target list. After detecting the target stimulus, the focus was moved to the other position. In the PU condition, the subjects used a wide focus that encompassed both positions, a focus that was larger the greater the distance between the positions. As is predicted from the zoom lens model, in the PK condition, there was no effect of distance. On the other hand, in the PU condition, there was an effect of the distance due to the inverse relation between the size of the focus and the attentional facilitation. This effect was reflected in the interaction of certainty and distance.

The fact that we found no effect of distance in the PK condition (nor even the expected ordering of the means) is surprising, because, in this experiment, the different distances were associated with different eccentricities. One possibility is that, as a consequence of the difficulty of the task, the cost of increasing eccentricity was relatively small and marginal in practical terms. This lack of effect can hardly be attributed to a Type II error, given that this is not the first time it has been found in this kind of task (A. Reeves, personal communication, May 23, 1996; Sperling \& Weichselgartner, 1995).

Whereas the results are easily accounted for by the zoom lens model, they cannot be explained by the spotlight model with analog movement (Egly \& Homa, 1991; Shulman et al., 1979; Tsal, 1983). However, there are two alternative explanations for the main result. First, it can be explained from a gradient model (Downing \& Pinker, 1985; Shulman et al., 1985), if different slopes are assumed as a function of the spatial position where attention is centered. Second, it can also be accounted for by a capacity allocation model (Shaw, 1978; Shaw \& Shaw, 1977 ) or by a hemifield model (Hughes \& Zimba, 1985, 1987), if a dependence of the amount of resources on eccentricity is assumed. We explored these alternatives in Experiments 2A and 2B.

\section{EXPERIMENTS 2A AND 2B}

The goal of these two experiments was to replicate the results of Experiment 1, eliminating any effect of retinal eccentricity and discarding the alternative explanations to our results mentioned above.

As mentioned in previous research, a main methodological problem in the field of visual attention is the confusion between the effect of distance and the effect of retinal eccentricity (Eriksen \& Murphy, 1987; Van der Heijden, 1992). It is clear that, in Experiment 1, these two variables varied jointly, although in a sense they could be separated by studying the interaction between distance and spatial certainty. However, we could state an alternative interpretation of the interaction found in Experiment 1 with a model such as that of Shaw and Shaw (1977) or from the hemifield model (Hughes \& Zimba, $1985,1987)$. In short, if attentional resources in the PU condition are distributed between the positions where the lists are presented, then a larger effect of retinal eccentricity as attentional resources decrease (i.e., an interaction between resources allocated and eccentricity) could account for our results. In order to discard this alternative explanation, we designed Experiments $2 \mathrm{~A}$ and $2 \mathrm{~B}$, basically similar to Experiment 1 but with all locations having the same retinal eccentricity.

As was pointed out in the previous section, gradients with different slopes centered in different positions could account for the interaction between spatial certainty and distance; however, if this explanation is correct, we should also find an effect of the distance in the PK condition. Although in these experiments we cannot derive from this model differential predictions of the effect of the distance as a function of spatial certainty, new evidence showing a lack of effect of the distance in the PK condition would help to discard this alternative interpretation.

Given that, in these experiments, the retinal eccentricity for the different distance values was constant, the zoom lens model predicts no dependence of the ASOR on the distance in the PK condition, since the subjects 


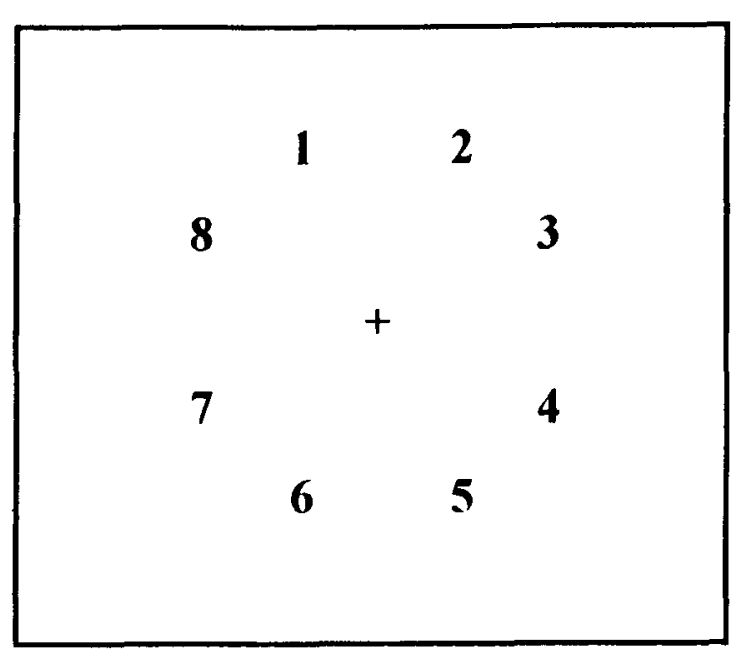

Figure 3. Positions where the lists were presented in Experiment 2.

would apply a small focus over the position where the target list was presented, and, as soon as the target was detected, the focus would be moved to the position where the response list was presented. In the PU condition, the subjects were forced to attend to both positions simultaneously in order to detect the target. Then, when the distance between the positions was increased, the size of the focus also increased, so that a later ASOR would be expected. Thus, from the zoom lens model, an effect of the interaction between spatial certainty and distance on the ASOR is again predicted.

\section{Method}

Subjects. Two groups of 8 subjects participated, one group for each experiment. The subjects were undergraduate students from the Universidad Autónoma de Madrid, with normal or corrected-tonormal vision.

Apparatus and Stimuli. The experimental setup was the same as in Experiment 1. Thirty-five of the list pairs designed for the Experiment 1,5 of them without the target, were selected for the Experiments $2 \mathrm{~A}$ and $2 \mathrm{~B}$. A circle and a square were used to signal the positions for the presentation of the lists.

Procedure. The procedure was similar for Experiments 2A and 2B. The variables manipulated in both experiments were spatial certainty, with two conditions (PK and PU), and the distance between the series $\left(1.24^{\circ}\right.$ and $2.67^{\circ}$ of visual angle). The designs were factorial $2 \times 2$ within subject. At the begining of each trial, a fixation point was presented at the center of an imaginary rectangle, and two marks indicated the positions where the lists were to be presented. An imaginary rectangle, with the longer axis in the vertical direction (vertical rectangle) was used for Experiment $2 \mathrm{~A}$, and a rectangle with the longer axis in the horizontal direction (horizontal rectangle) for Experiment 2B. This was the only difference between Experiments $2 \mathrm{~A}$ and $2 \mathrm{~B} .{ }^{2}$ For Experiment $2 \mathrm{~A}$, in the short distance condition $\left(1.24^{\circ}\right.$ between the centers of the stimuli), the lists could be presented either in the upper vertices of the vertical imaginary rectangle or in the lower vertices (Figure 3, Positions 1 and 2 or Positions 5 and 6$)$; in the large distance condition $\left(2.67^{\circ}\right.$ between the centers of the stimuli), the possible locations of the series were the upper and lower corners, at the right or left of the vertical imaginary rectangle (Figure 3, Positions 1 and 6 or Positions 2 and 5). In Experiment $2 \mathrm{~B}$, the imaginary rectangle was horizontal. In short distance condition, the list could be presented either in the vertices at the right or left of the fixation point (Figure 3, Positions 3 and 4 or Positions 7 and 8); in the large distance condition, the positions above or below the fixation were used (Figure 3, Positions 8 and 3 or Positions 7 and 4). In both experiments, in the PK condition, the marks that were presented along with the fixation point before each trial were always a circle and a square. The circle indicated the position where the target list was to be presented, and the square indicated the position where the response list was to be presented. In the PU condition, the marks were two squares. All positions had a constant retinal eccentricity of $1.47^{\circ}$.

Two blocks of 70 trials each were prepared, one for each distance; 10 trials from each block did not include the target. In half of the trials, the lists were presented in two of the possible positions; in the other half, they were presented in the remaining positions. The subjects ran four blocks, two for each condition of certainty and one for each distance, in a single experimental session of about $1 \mathrm{~h}$. The order of presentation of the conditions of spatial certainty was balanced. Within each condition of spatial certainty, the order of presentation of the conditions of distance was also balanced. Before running the two blocks of each condition of spatial certainty, the subjects received a practice block of that condition, formed by 35 trials with the short distance and 35 with the large distance. The eye movements were again monitored by means of a video camera, and the subjects who showed obvious movements were excluded from the experiments (only 1 was excluded). The same dependent variable as in Experiment 1 was analyzed.

\section{Results and Discussion}

The collapsed data (see note 2 ) were again analyzed by means of a repeated measures $2 \times 2$ (spatial certainty $\times$ distance) ANOVA.

In the PK condition, the mean values of the ASOR were 3.06 and 2.95 for the distances short and large, respectively. In the PU condition, the values were 3.27 and 3.80 , respectively. In the ANOVA, main effect of spatial certainty reached significance $[F(1,15)=18.85, p<.001]$, as did the effect of distance $[F(1,15)=6.92, p<.02]$. We also found a significant interaction between spatial certainty and distance $[F(1,15)=16.46, p<.001]$, as can be observed in Figure 4 (the ASOR in this figure has been transformed to milliseconds, one position equaling $116 \mathrm{msec}$ ). For the PK condition, there was no significant difference between the two conditions of distance (Tukey test, $p>.05$ ); for the PU condition, the ASOR for the large distance was significantly greater than that for the short distance (Tukey test, $p<.001$ ).

The pattern of results in Experiment 2 replicates the main result of Experiment 1: the interaction between spatial certainty and distance. One of the goals of Experiment 2 was to discard some alternative explanations to that offered by the zoom lens model of the interaction between spatial certainty and distance found in Experiment 1 . We had pointed out that, from a capacity allocation model (Shaw, 1978; Shaw \& Shaw, 1977) or from a hemifield model (Hughes \& Zimba, 1985, 1987), this interaction could be explained by an interaction between the amount of resources and retinal eccentricity. The evidence found in Experiment 2, in which retinal eccentricity was controlled, go against that explanation. 


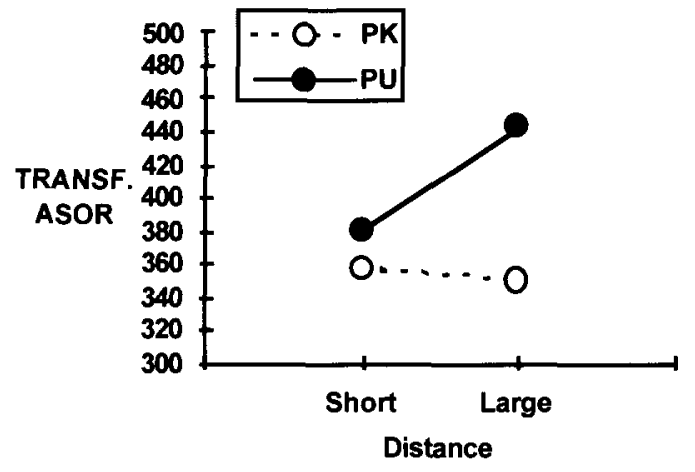

Figure 4. Average serial order of responses (ASOR; in milliseconds) for Experiment 2 ( 1 item $=116 \mathrm{msec})$.

We had also pointed out that, from the gradient model (Downing \& Pinker, 1985), under some specific circumstances, this interaction could also appear. However, from that model, there would necessarily be an effect of distance in the PK condition, a prediction contrary to the predictions of the zoom lens model. Again, as in Experiment 1 , there was no effect of distance in the PK condition.

\section{GENERAL DISCUSSION}

Overall, the results of this series of experiments match well with the predictions derived from the zoom lens model. The importance of this conclusion resides not only in the matching between the data and the model but, above all, in the fact that the results were obtained with an experimental paradigm different from that customarily used to test the model.

However, we should discuss in more detail the mechanisms by which the observed effects are produced: They can be produced in three different stages of each trial: (1) the detection of the T, (2) the possible changes in attentional distribution after detecting the $T$, and (3) the processing and/or selection of a stimulus from the response list. If subjects follow the experimental instructions (as encouraged to by the structure of the task), then the bulk of the observed effects can be produced in the first stage, corresponding to the detection of the $T$. This is because, in the PK condition, for all the distances used, the detection stage is accomplished with a small focus, whereas in the PU condition, the focus is greater when the distance between positions is increased. Let us suppose that, in all certainty and distance conditions, the second stage mentioned above remains the same: After detecting the $T$, a small focus is built on the position where the response list appears, and the process of identifying an item of the response list is executed always with that small focus. Let us also suppose that the time necessary to build up this small focus depends neither on the size nor on the distance of its previous position, so that the distance is not in itself the cause of the observed differences. In this case, the observed effect would have to be attributed exclusively to the differences in the time in- vested in detection of the T. Reeves and Sperling (1986, p. 184; see Figure 3) found that the items reported from the response list tended to originate from somewhat earlier serial positions when the target was a square than when it was a letter. We believe that this was due to the fact that detecting a square is accomplished more rapidly than recognizing a letter. Identifying the targets $C$ and $U$ delays the beginning of the process for closing the first focus. Consequently, the reported items originate, on average, in subsequent positions. Basically, the effect is the same as that the one we have obtained, by producing different times in the detection of the T. Thus, it is possible that an explanation of the effects we have observed can be based exclusively on the detection of the $T$. It can be argued that, in the PU condition, subjects must make a decision (where the $\mathrm{T}$ has been presented) that does not have to be made in the PK condition; this decision can add on extra time, but it should be constant across the distance conditions. Thus, even accepting this idea would leave unexplained the interaction-that is, the different effect of distance in the PU and PK conditions.

Let us now suppose that the time invested in the second stage is not the same in all conditions. There is evidence enough to support the idea that the time necessary to shift a small focus of attention is not proportional to the distance covered, evidence originating from various experimental paradigms (Eriksen \& Webb, 1989; Kwak, Dagenbach, \& Egeth, 1991; Murphy \& Eriksen, 1987; Sagi \& Julesz, 1985; Sperling \& Weichselgartner, 1995; Weichselgartner, Sperling, \& Reeves, 1985). Furthermore, our own results do not reveal effects of distance in the PK condition. Also, we have positive evidence of the fact that a zooming process needs more time than a shifting one (Stoffer, 1993); however, this effect would merely add a constant time, and the interaction between spatial certainty and distance would remain unexplained. On the other hand, if this last point were correct, then we would have to find a difference between the short distance conditions, since, in the PU condition, the detection of the T must be slower and, furthermore, must be produced by a zooming instead of a shifting. However, we have not found a difference between these conditions.

A more credible possibility is that, in the PK condition, subjects shift the focus once the $T$ is detected, whereas in the PU condition, they adopt a broad focus that encompasses both positions and is maintained during the trial. If this were true, we would have to consider the consequences of the fact that the stage of processing and selecting an item from the response list is accomplished with foci of different sizes. Reeves and Sperling (1986) have demonstrated that, although in this task the items are correctly identified, as the task is accomplished in nonoptimal attentional conditions, the order information associated with each item is partially lost. As less attention is paid, more order information is lost; the more order information is lost, the later is the average item reported. Thus, since our experimental manipulations may have meant that, in the PU condition, there is a decrease in the attention paid to the processing of the response list 
as the distance is increased, there must be an additional effect of the distance associated with processing of the items in the response list. This may also explain why there are more trials without a response in the PU conditions. As they are accomplished with less attention, and more order information is lost, subjects feel less confident and more often give an "I don't know" response.

A different logical possibility is that, in both conditions of spatial uncertainty subjects have been using a broad focus that encompasses both lists; however, we should reject this, since the interaction would remain unexplained.

In short, from our point of view, the differences observed in the dependent variable ASOR do not only reveal differences in attention shifting. We believe that the observed pattern of results was due to (1) differences in the time needed to detect the T, (2) (probably) a constant time added only to the PK conditions due to the time needed to shift the small focus, which is independent of the distance, and (3) (probably) an effect due to a differential loss of the order information from the response list in the PU conditions (this loss was also greater with increasing distance between the locations). Thus, the observed interaction effect was wholly due to attentional factors - that is, the different levels of performance were due to different amounts of attention applied to the detection of the $T$, as well as to the processing of the response list. Factor 2 above was responsible for the absence of differences between the short distance conditions, since it compensated for the other factors that favored the PK conditions.

One of the assumptions of the zoom lens model is the unity of the attended area. A main source of evidence in favor of the contiguity of the attended area comes from experiments in which the identity of the distractors presented in the area between two targets has an effect (e.g., Pan \& Eriksen, 1993). However, Kramer and Hahn (1995) have suggested that, in these demonstrations, the distractors can attract attention automatically due to their abrupt onset. In Kramer and Hahn's experiments, the abrupt-onset character of the distractors was eliminated, and their effect disappeared. Taking into account these results, we cannot discard that in the PU condition, subjects use two separate foci; one centered on the target list, and the other centered on the response list. However, given that in the present experiments, we found an effect of distance in the PU condition, the interesting and challenging conclusion would be that to attend to two separate objects would demand greater effort as the distance between them increases. A possible explanation for thisthough at a speculative level--is that subjects must inhibit the greater area between them.

Yet the issue remains of why an effect of distance has been found in some experiments but not in others. The patterns of results in our experiments can serve as a basis for a proposal to solve these conflicting results. Our proposal is that the apparent distance effects reported in several experiments are not authentic attentional shiftings; rather, they are due to the use of foci of different size in the different experimental conditions or to attentional disposi- tions such as gradients, but without real shifts of attention. On the other hand, experiments in which there has not been an effect of distance have used a broad focus, always of the same size (thus, again without a real attentional movement), or a small focus that changes its position; the time necessary for this shift, however, would be independent of the distance between the initial and final positions. The episodic theory (Sperling \& Weichselgartner, 1995), a formal and much more sophisticated model than the zoom lens model, arrives at the same basic conclusions as the zoom lens model when it is applied to a small focus that is moved from one position to another, but it has not yet been developed for situations in which the size of the attended area is manipulated, either by the instructions and/or by the task itself, as was the case in the present experiments.

\section{REFERENCES}

BARRIOPEDRO, M. I. (1994, May). Mapping the allocation of spatial attention with an RSVP task. Poster presented at the conference Converging Operations in the Study of Visual Selective Attention, Beckman Institute, University of Illinois at Urbana-Champaign.

Botella, J., \& Eriksen, C. W. (1992). Filtering versus parallel processing in RSVP tasks. Perception \& Psychophysics, 51, 334-343.

Botella, J., Garcia, M. L., \& Barriopedro, M. [I.] (1992). Intrusion patterns in rapid serial visual presentation tasks with two response dimensions. Perception \& Psychophysics, 52, 547-552.

DownING, C. J. (1988). Expectancy and visual spatial attention: Effects on perceptual quality. Journal of Experimental Psychology: Human Perception \& Performance, 14, 188-202.

Downing, C. J., \& PinkeR, S. (1985). The spatial structure of visual attention. In M. I. Posner \& O. S. M. Marin (Eds.), Attention and performance $X I$ (pp. 171-187). Hillsdale, NJ: Erlbaum.

EGLY, R., \& HomA, D. (1991). Reallocation of visual attention. Journal of Experimental Psychology: Human Perception \& Performance, 17, 142-159.

ERIKSEN, C. W. (1990). Attentional search of the visual field. In D. Brogan (Ed.), Visual search (pp. 3-19). New York: Taylor \& Francis.

ERIKSEN, C. W., \& HoffMAN, J. E. (1972). Temporal and spatial characteristics of selective encoding from visual displays. Perception \& Psychophysics, 12, 201-204.

Eriksen, C. W., \& MurPhy, T. D. (1987). Movement of attentional focus across the visual field: A critical look at the evidence. Perception \& Psychophysics, 42, 299-305.

Eriksen, C. W., PAn, K., \& Botella, J. (1993). Attentional distribution in visual space. Psychological Research, 56, 5-13.

Eriksen, C. W., \& Rohrbaugh, J. W. (1970). Some factors determining efficiency of selective attention. American Journal of Psychology, 83, 330-342.

ERIKSEN, C. W., \& SPENCER, T. (1969). Rate of information processing in visual perception: Some results and methodological considerations. Journal of Experimental Psychology Monographs, 79(2, Pt. 2), 1-16.

ERIKSEN, C. W., \& ST. JAMES, J. D. (1986). Visual attention within and around the field of focal attention: A zoom lens model. Perception \& Psychophysics, 40, 225-240.

ERIKSEN, C. W., \& WeBB, J. M. (1989). Shifting of attentional focus within and about a visual display. Perception \& Psychophysics, 45, 175-183.

ERIKSEN, C. W., \& YEH, Y. Y. (1985). Allocation of attention in the visual field. Journal of Experimental Psychology: Human Perception \& Performance, 5, 583-597.

Gathercole, S. E., \& Broadbent, D. E. (1984). Combining attributes in specified and categorized target search: Further evidence for strategy differences. Memory \& Cognition, 12, 329-337.

Hughes, H. C., \& Zimba, L. D. (1985). Spatial maps of directed visual attention. Journal of Experimental Psychology: Human Perception \& Performance, 11, 409-430. 
Hughes, H. C., \& ZimBa, L. D. (1987). Natural boundaries for the spatial spread of directed visual attention. Neuropsychologia, 25, 5-18.

JoNides, J. (1980). Toward a model of the mind's eye. Canadian Journal of Psychology, 34, 103-112.

JONIDES, J. (1983). Further toward a model of the mind's eye's movement. Bulletin of the Psychonomic Society, 21, 247-250.

KANWISHER, N. G. (1987). Repetition blindness: Type recognition without token individuation. Cognition, 27, 117-143.

KLEIN, R., \& MCCoRmick, P. (1989). Covert visual orienting: Hemifieldactivation can be mimicked by zoom lens and midlocation placement strategies. Acta Psychologica, 70, 235-250.

Kramer, A. F., \& Hahn, S. (1995). Distribution of attention over noncontiguous regions of the visual field. Psychological Science, 6, 381386.

Kwak, H.-W., Dagenbach, D., \& Egeth, H. (1991). Further evidence for a time-independent shift of the focus of attention. Perception $\&$ Psychophysics, 49, 473-480.

LABERGE, D. (1983). Spatial extent of attention to letters in words. Journal of Experimental Psychology: Human Perception \& Performance, 9, 371-379.

LABERGE, D., \& Brown, V. (1986). Variations in size of the visual field in which targets are presented: An attentional range effect. Perception \& Psychophysics, 40, 188-200.

LAWRENCE, D. H. (1971). Two studies of visual search for word targets with controlled rates of presentation. Perception \& Psychophysics, 10, 85-89.

MCCORMICK, P. A., \& KLEIN, R. (1990). The spatial distribution of attention during covert visual orienting. Acta Psychologica, 75, 225-242.

McLean, J. P., Broadbent, D. E., \& Broadbent, M. H. P. (1983). Combining attributes in rapid sequential visual presentation tasks. Quarterly Journal of Experimental Psychology, 22, 279-287.

MURPHY, T. D., \& ERIKSEN, C. W. (1987). Temporal changes in the distribution of attention in the visual field in response to precues. Perception \& Psychophysics, 42, 576-586.

PAN, K., \& ERIKSEN, C. W. (1993). Attentional distribution in the visual field during same-different judgments as assessed by response competition. Perception \& Psychophysics, 53, 134-144.

POSNER, M. I. (1978). Chronometric exploration of the mind. Hillsdale, NJ: Erlbaum.

PoSNER, M. I. (1980). Orienting of attention. Quarterly Journal of Experimental Psychology, 32, 3-25.

Posner, M. I., Nissen, M. J., \& Ogden, W. C. (1978). Attended and unattended processing modes: the role of set for spatial location. In H. L. Pick \& I. J. Saltzman (Eds.), Modes of perceiving and processing information (pp. 137-157). Hillsdale, NJ: Erlbaum.

Posner, M. I., SNyder, C. R. R., \& Davidson, B. J. (1980). Attention and the detection of signals. Journal of Experimental Psychology: General, 109, 160-174.

Raymond, J. E., Shapiro, K. L., \& ARnell, K. M. (1992). Temporary suppression of visual processing in a RSVP task: An attentional blink? Journal of Experimental Psychology: Human Perception \& Performance, 18, 849-860.

ReEvES, A., \& SPERLING, G. (1986). Attention gating in short-term visual memory. Psychological Review, 93, 180-206.

SAGI, D., \& Julesz, B. (1985). Fast, noninertial shifts of attention. Spatial Vision, 1, 141-149.

Shapiro, K. L., RAymond, J. E., \& ARnell, K. M. (1994). Attention to visual pattern information produces the attentional blink in rapid serial visual presentation. Journal of Experimental Psychology: Human Perception \& Performance, 20, 357-371.

SHAw, M. L. (1978). Temporal changes in the distribution of attention in the visual field in response to precues. Journal of Experimental Psychology: Human Perception \& Performance, 4, 586-598.

Shaw, M. L., \& Shaw, P. (1977). Optimal allocation of cognitive resources to spatial locations. Journal of Experimental Psychology: Human Perception \& Performance, 3, 201-211.

Shulman, G. L., Remington, R. W., \& McLean, J. P. (1979). Moving attention through visual space. Journal of Experimental Psychology: Human Perception \& Performance, 5, 522-526.
Shulman, G. L., Sheehy, J. B., \& Wilson, J. (1986). Gradients of spatial attention. Acta Psychologica, 61, 167-181.

Shulman, G. L., Wilson, J., \& Sheehy, J. B. (1985). Spatial determinants of the distribution of attention. Perception \& Psychophysics, 37, 59-65.

SPERling, G., \& Reeves, A. (1980). Measuring the reaction time of an unobservable response: a shift of visual attention. In R. Nickerson (Ed.), Attention and performance VIII (pp. 347-360). Hillsdale, NJ: Erlbaum

SPERling, G., \& Weichselgartner, E. (1995). Episodic theory of the dynamics of spatial attention. Psychological Review, 102, 503-532.

STOFFER, T. H. (1993). The time course of attentional zooming: A comparison of voluntary and involuntary allocation of attention to the levels of compound stimuli. Psychological Research, 56, 14-25.

Theeuwes, J. (1995). Perceptual selectivity for color and form: On the nature of the interference effect. In A. F. Kramer, M. G. H. Coles, \& G. D. Logan (Eds.), Converging operations in the study of visual selective attention (pp. 297-314). Washington, DC: American Psychological Association.

TsaL, Y. (1983). Movements of attention across the visual field. Journal of Experimental Psychology: Human Perception \& Performance, 9, 523-530.

VAN DER HEIJDEN, A. H. C. (1992). Selective attention in vision. London: Routledge.

Weichselgartner, E., Sperling, G., \& ReEves, A. (1985). Effects of concurrent tasks, distance, and visual obstacles on shifts of visual attention. Proceedings of the Eastern Psychological Association, 56, 72.

YANTIS, S. (1988). On analog movements of visual attention. Perception \& Psychophysics, 43, 203-206.

YANTIS, S. (1995). Attentional Capture in Vision. In A. F. Kramer, M. G. H. Coles, \& G. D. Logan (Eds.), Converging operations in the study of visual selective attention (pp. 45-76). Washington, DC: American Psychological Association.

YANTIS, S., \& Johnston, D. N. (1990). Mechanisms of attentional priority. Journal of Experimental Psychology: Human Perception \& Performance, 16, 812-825.

YANTIS, S., \& JonES, E. (1991). Mechanisms of attentional selection: Temporally modulated priority tags. Perception \& Psychophysics, 50, 166-178.

YANTIS, S., \& JoNIDES, J. (1984). Abrupt visual onsets and selective attention: Evidence from visual search. Journal of Experimental Psychology: Human Perception \& Performance, 10, 601-621.

YANTIS, S., \& JoNidEs, J. (1990). Abrupt visual onsets and selective attention: Voluntary versus automatic allocation. Journal of Experimental Psychology: Human Perception \& Performance, 16, 121-134.

\section{NOTES}

1. The terms attention response time used by Sperling and Reeves (1980; Reeves \& Sperling, 1986) and response time would be inappropriate here, because these terms prejudge the nature of any effect eventually observed. We prefer the descriptive label average serial order of the responses. We deal with this point in the General Discussion section.

2 . We initially used only a vertical imaginary rectangle (Experiment $2 \mathrm{~A}$ ). However, the reviewers pointed out that a hemifield model could explain the results; therefore, we decided to replicate the experiment, placing the stimuli in a horizontal imaginary rectangle, in order to eliminate possible confusion between distance and hemifield (Experiment $2 \mathrm{~B}$ ). The results of Experiment $2 \mathrm{~B}$ were identical to those of Experiment 2A; on analysis of the data with an ANOVA (including an experiment factor), no main effects or interactions with other variables were shown. Therefore, the analysis was carried out with collapsed data.

(Manuscript received January 3, 1996; revision accepted for publication November 11, 1997.) 